\title{
Application of CSHA Frailty Index and Clinical Frailty Scale in Geriatric Assessment of Elderly Males in China
}

\author{
Liyun Chu*, Chunhua Shi \\ Department of Gerontology, The 971 Hospital of Chinese PLA, Qingdao, China \\ Email address: \\ chuliyun555@126.com (Liyun Chu),sch5389@163.com (Chunhua Shi) \\ ${ }^{*}$ Corresponding author
}

To cite this article:

Liyun Chu, Chunhua Shi. Application of CSHA Frailty Index and Clinical Frailty Scale in Geriatric Assessment of Elderly Males in China. American Journal of Nursing Science. Vol. 9, No. 4, 2020, pp. 235-238. doi: 10.11648/j.ajns.20200904.22

Received: June 3, 2020; Accepted: June 22, 2020; Published: June 28, 2020

\begin{abstract}
People at an advanced age often live with multiple chronic diseases as well as disabilities. To access the health condition of elderly persons, various models have been used in developed countries in the West. However, it is not known how these models apply to the elderly in Asian developing countries. This study investigated the application of the Clinical Frailty Scale-09, a widely used method in patient assessment in Western countries, to older adults living in mainland China. Two hundred and ten elderly males were assessed for their health conditions using a list of variables by the Canadian Study of Health and Aging to construct the 70-item CSHA Frailty Index. The obtained Frailty Indices were compared with the scores of The Clinical Frailty Scale-09 for the same sample group. The assessment revealed the changing pattern in the health condition of the sampled population. Compared to the group aged 65-74 years old, the Frailty Index and the Clinical Frailty Scale increased in the groups aged 75-84 and 85-89 years old. The greatest increase was in the group aged $\geq 90$ years old. The scores of the Frailty Index and the Clinical Frailty Scale- 09 correlate with each other. These findings suggest that the Frailty Index and Clinical Frailty Scale-09 provide reliable assessment of the health condition of elderly Chinese males.
\end{abstract}

Keywords: Geriatric Medicine, CSHA Frailty Index, The Clinical Frailty Scale-09, Comprehensive Geriatric Assessment

\section{Introduction}

In the practice of geriatric medicine, it is critical to know patients' complex medical and social needs in order to make the right decisions for them. The challenge is that with the increase in age, elderly patients get more and more susceptible to various diseases and less and less responsive to treatments. In fact, almost all adult-onset illnesses become more common with age. Many elderly people have multiple diseases and other health problems that do not reach the level of disease $[1,2]$. Therefore, treatment of diseases of elderly patients should take into account both patients' overall background, as well as their comorbidities.

A long-standing issue in the geriatric medical field has been the ineffective methodology available to measure the aging-related health conditions of the elderly. The Canadian geriatrician professors Rockwood and Mitnitski created an aging model based on health-defect accumulations, thus providing a reasonable and effective way to solve the problem of quantization of the health status of the elderly [3, 4]. The model has been well-studied, used for 33,000 surveys in Canada, Australia, the United States, and Sweden, which has confirmed the effectiveness of this evaluation method [4 7]. However, it is not known whether the model applies to aging populations in Asian developing countries.

We conducted a survey of 210 males over 65 years of age in mainland China using the aging model of Rockwood and Mitnitski [4] to see whether the model applies to a Chinese population. Our study made full use of data collected from general health surveys, including symptoms, medical history, functional impairments, mental state, and capacity for action. The various health defects in aging were quantified, and mathematical methods were applied to identify the information contained. The results show that the evaluation method is feasible for Chinese men and can be widely used in the clinical work of geriatric medicine in China. 


\section{Method}

\subsection{Participants}

With the approval of the Ethical Committee of the 971 Hospital of Chinese PLA, this survey was conducted following the principle of random sampling: all males aged 65 or older coming for regular physical examinations and willing to join the study were included. Two hundred and ten such males recruited from five different Cadre's Sanitariums in Qingdao, China, were set as the subjects. They were aged from 65 to 92 years old with an average age of $81.5 \pm 3.9$ years; all were retired veterans in Cadre's Sanitariums. Seventy-one subjects were in the group aged 65 to 74 years old; 87 subjects were in the group aged 75 to 84 years old; 49 subjects were in the group aged 85 to 89 years old; and three were in the group aged 90 years old and above. In the process of data collection, face-to-face interviews were conducted individually in Cadre's Sanitariums in Qingdao, China, from January to December 2018.

\subsection{Data Collection}

(1) We collected a list of variables used by the Canadian Study of Health and Aging to construct the 70-item CSHA Frailty Index as follows: 1 changes in activities of daily life, 2 head and neck problems, 3 neck muscle tension, 4 facial muscle tension, 5 difficulties in wearing clothing, 6 difficulties in bathing, 7 difficulties in barbering, 8 incontinence, 9 difficulty going to the toilet, 10 reduced muscle volume, 11 rectal lesions, 12 gastrointestinal problems, 13 difficulties in cooking, 14 difficulties in sucking, 15 difficulties going out alone, 16 damaged movement function, 17 cognitive impairments with family history, 18 skin diseases, 19 malignant disease, 20 breast disease, 21 celiac disease, 22 pouting reflection, 23 palm chin reflection, 24 history of other diseases, 25 emotional problems, 26 feeling sad or depressed, 27 history of depression, 28 feeling tired, 29 depression, 30 sleep changes, 31 restlessness, 32 deterioration of the memory, 33 shortterm memory impairment, 34 long-term memory impairment, 35 mental function change, 36 cognitive impairment, 37 delirium, 38 paranoia, 39 history of cognitive disorder, 40 history of thyroid disease, 41 thyroid disease, 42 limbs and muscle tension, 43 limbs coordination disorder, 44 body coordination disorder, 45 difficulty in standing, 46 irregular gait, 47 fell down, 48 local epileptic seizure, 49 generalized epileptic seizures, 50 syncope or transient amaurosis, 51 headache, 52 cerebrovascular disease, 53 history of stroke, 54 history of diabetes, 55 hypertension, 56 peripheral vascular pulsation, 57 heart disease, 58 myocardial infarction, 59 arrhythmia, 60 congestive heart failure, 61 lung disease, 62 respiratory diseases, 63 musculoskeletal problems, 64 slow limbs motion, 65 damaged balance, 66 resting tremor, 67 postural tremor, 68 intention tremor, 69 medical history of Parkinson's disease, 70 history of degenerative disease. The Frailty Indices were calculated from these 70 kinds of health defects listed above. For instance, if a particular individual has 14 health defects, then its weakness index is $14 / 70=0.2[4,6,8]$.

(2) A quantitative description of the weakness in the elderly was adopted from Rockwood and Mitnitski. The Clinical Frailty Scale-09 has nine levels: Level 1 (very healthy) - physically strong, positive and active, energetic and vibrant, undertakes regular physical exercise, and in the most healthy state of the age group; Level 2 (healthy) - has no obvious symptoms of disease but not as good as Level 1 , takes regular physical exercise, and very active occasionally; Level 3 (maintains health) - health defects can be controlled, but besides regular walking, there is no regular physical exercise; Level 4 (weak and easily injured) - does not need help from others in daily life but some of physical symptoms limit daily activities; 5 (mild weak) - shows slow movement and help is required in activities with the tools of daily living (e.g., going to the bank, taking the bus, doing heavy housework, and taking medicine); Level 6 (moderately weak) - help is required in all outdoor activities, climbing the stairs in the house and taking a bath; Level 7 (severely weak) totally incapable of taking care of oneself, but the body is relatively stable and there is no risk of death over a period of time ( $<6$ months); Level 8 (very seriously weak) - totally incapable of taking care of oneself, close to the end of life, and cannot recover from a disease; Level 9 (end-stage) dying and near the end of life, and survival time is less than six months [9].

\subsection{Statistical Analysis}

The SPSS13.0 statistical software package was used for statistical analysis. Group data are shown in mean and standard deviation $(\bar{x} \pm S D)$, and comparisons of means were subject to the $t$-test. Correlation of group data was analyzed by calculating the bivariate linear correlation coefficient.

\section{Results}

\subsection{Frailty Index of Elderly Males in Age Groups}

We first determined the Frailty Index of 210 male subjects in four age groups using the 70 variables of the Canadian Study of Health and Aging, which were same as those used by Rockwood et al. [9]. The means and standard deviations $(\bar{x} \pm S D)$ of the Frailty Index for each group are listed in Table 1. The group of subjects 65-74 years old scored $0.15 \pm$ 0.04 , indicating that their conditions were "well but with treated comorbid disease" according to Rockwood et al. [9]. The group aged 75-84 years old scored $0.18 \pm 0.05$, which was a significant increase compared to the group aged 65-74 years old $(t=2.01, p<0.05)$. The mean Frailty Index of the next group aged 85-89 years old was $0.19 \pm 0.05$, which is also significantly higher than that of the group aged 65-74 years old $(t=3.43, p<0.05)$, but not significantly different from the index of the group of subjects aged 75 to 84 years old. The group of the most advanced age $(\geq 90)$ had only three subjects, and this group had a significantly higher mean Frailty Index $(0.22 \pm 0.07)$ compared to both groups aged 65 - 
74 and 75-84 years old $(t=3.19, p<0.05 ; t=2.33, p<0.05$, respectively). The data clearly indicates that the Frailty Index gradually increases with an increase in age.

Table 1. Frailty Index (x-SD) of 210 elderly men above 65 years old.

\begin{tabular}{lll}
\hline Groups (Age) & Case number & Frailty Index \\
\hline $65-74$ & 87 & $0.15 \pm 0.04$ \\
$75-84$ & 71 & $0.18 \pm 0.05 \mathrm{a}$ \\
$85-89$ & 49 & $0.19 \pm 0.05 \mathrm{a}$ \\
$\geq 90$ & 3 & $0.22 \pm 0.07 \mathrm{ab}$ \\
\hline
\end{tabular}

a: compared with group aged 65-74 years old, $p<0.05$.

b: compared with group aged 75-84 years old, $p<0.05$.

Compared with published data [6,9], the Frailty Indices of all age groups below 90 years old are comparable with the indices of the same ages in Western developed countries. In addition, our data show a smooth change in the Frailty Index over age groups. For example, the scale of the Frailty Index increase after the five years of 85-89 years old is at the same rate as the change in the previous 10 years. In other words, the observed Frailty Index changes do not have a dramatic increase in the speed of aging in our samples of subjects aged 85-89.

\subsection{Frailty Scale of Elderly Men in Age Groups}

We further assessed the Frailty Scale for each subject and calculated the mean Frailty Scale for each age group. As shown in Table 2, along with age increases, the Frailty Scale gradually increases. Compared with the group aged 65-74 years old, the Frailty Scale of the group aged 75-84 years old increased significantly $(t=3.55, p<0.05)$ from $2.1 \pm 0.5$ for the group aged $65-74$ years old to $3.3 \pm 0.7$ for the group aged 75-84 years old. The Frailty Index of the group over 90 years old is significantly higher than that of both groups aged 65-74 and 75-84 years old $(t=4.24, p<0.05 ; t=4.76, p<$ 0.05 , respectively).

Table 2. Frailty Scale $(\bar{x} \pm S D)$ of 210 elderly men above 65 years old.

\begin{tabular}{lll}
\hline Groups (Age) & Case number & Frailty Scale \\
\hline $65-74$ & 87 & $2.1 \pm 0.5$ \\
$75-84$ & 71 & $3.3 \pm 0.7 \mathrm{a}$ \\
$85-89$ & 49 & $3.7 \pm 0.9 \mathrm{a}$ \\
$\geq 90$ & 3 & $4.0 \pm 0.9 \mathrm{ab}$ \\
\hline
\end{tabular}

a: compared with group aged 65-74 years old, $p<0.05$.

b: compared with group aged 75-84 years old, $p<0.05$.

\subsection{Correlation Analysis of Group Frailty Index and Frailty Scale}

The Frailty Index and Frailty Scale used in this study assess different physical functions, yet both are designed to quantify human health conditions. However, for the aging population in China, will the measurements of the Frailty Index and Frailty Scale parameters agree with each other? With our data, we calculated the correlation between the Frailty Index and Frailty Scale data. The correlation analysis is summarized in Table 3. From our calculation of the data in the previous sections, the Frailty Index and Frailty Scale of the males over 65 years old show a significant positive correlation. Thus, the Frailty Index and Frailty Scale describe the health level of aged Chinese males in the same way.

Table 3. Correlation analysis (r) for Frailty Index and Frailty Scale.

\begin{tabular}{lllll}
\hline Age Group & $\mathbf{6 5} \sim \mathbf{7 4}$ & $\mathbf{7 5} \sim \mathbf{8 4}$ & $\mathbf{8 5} \sim \mathbf{8 9}$ & $\geq \mathbf{9 0}$ \\
\hline Cases & 87 & 71 & 49 & 3 \\
$\mathrm{r}$ & $0.63 \mathrm{a}$ & $0.58 \mathrm{a}$ & $0.61 \mathrm{a}$ & $0.49 \mathrm{~b}$ \\
\hline
\end{tabular}

$\mathrm{a}: p>0.01, \mathrm{~b}: p>0.05$

\section{Discussion}

We have been using the Frailty Index and Frailty Scale to access patients' health conditions. In practice, many definitions of frailty are used to assess the overall health conditions of elderly patients, both in clinical and epidemiological studies. Although no one definition has yet prove to be the best for all applications, frailty can be viewed as the risk state that comes about due to the accumulation of deficits [10 - 12]. We recognize these deficits clinically as symptoms, signs, laboratory abnormalities, diseases and disabilities. As such, the Rockwood definition of frailty is essentially a Cumulative Burden Index which includes health conditions and deficits [13 - 15]. The 70-item CSHA Frailty Index by Rockwood and Mitnitski just provided detailed explanations of frailty.

Asian countries are facing a rapid increase in their aging populations, which is connected to an accumulation of health problems in the populations. For the complexity in the health condition of each elderly individual, it is insufficient to just describe the disease as "atypical." Hence, we need a new methodology for describing a health state. The Frailty Index and Frailty Scale provide a useful model for Asian developing countries to consider. We found that adoption of well-established techniques is a quick way to develop our own methods of newly developed branches of medicine. Our study showed that the Frailty Index and Frailty Scale faithfully depict the health condition of males in China; thus, they will provide reliable assessments for aging patients. The results also indicate that the aging process of different ethnic groups in Chinese urban areas and in the Western developed countries may be similar.

\section{Conclusion}

This study revealed that for Chinese men over the age of 65, both the Frailty Index and Frailty Scale increase gradually along with the increase in age. Our observation is similar to Mitnitski's report [6,9], and the Frailty Index and Frailty Scale from our study show a significant positive correlation with each other. Therefore, the CSHA Frailty Index and the Clinical Frailty Scale-09 can be adopted to Chinese populations in the future.

Our data lend support to developing suitable nursing interventions for aging populations. We believe that the application of the Frailty Index and Frailty Scale will provide a useful model in the accurate description of a patient's 
condition and that the further improvement of such application is bound to be more effective in clinical practices caring for elderly people.

\section{References}

[1] Mitnitski A., Song X. and Rockwood K. (2007). Improvement and decline in health status from late middle age: modeling age-related changes in deficit accumulation. Exp Gerontol, 42 (11), 1109-1115.

[2] Rothman M. D., Leo-Summers L. and Gill T. M. (2008). Prognostic significance of potential frailty criteria. J Am Geriatr Soc, 56, 2211-2216.

[3] Mitnitski A., Mogilner A. J., and Rockwood K. (2001). Accumulation of deficits as a procy measure of aging. Scientific world journal, 8, 323-326.

[4] Rockwood K., Song X., MacKnight C., Bergman H., Hogan D. B., McDowell I. and Mitnitski A. (2005). A global clinical measure of fitness and frailty in elderly people. Canadian Medical Association journal, 173 (5), 489-495.

[5] Kojima G., Iliffe S. and Walters K. (2018). Frailty index as a predictor of mortality: a systematic review and meta-analysis. Age and Ageing, 47 (2), 193-200.

[6] Mitnitski A., Song X., Skoog I., Broe G. A., Cox J. L., Grunfeld E. and Rockwood K. (2005). Relative fitness and frailty of elderly men and women in developed countries and their relationship with mortality. J Am Geriatr Soc, 53, 21842189.
[7] Rockwood K. and Mitnitski A. (2007). Frailty in relation to the accumulation of deficits. J Gerontol A Biol Sci Med Sci, $62,722-727$.

[8] Searle S. D., Mitnitski A., Gahbauer E. A., Gill T. M. and Rockwood K. (2008). A standard procedure for creating a frailty index. BMC Geriatr, 8, 24.

[9] Rockwood K., Song X., Mitnitski A. and Yu P. (2009). Geriatric medicine and the care of frail elderly People. Chinese Journal of Geriatrics, 28, 353-365.

[10] Kim D. H., Schneeweiss S., Glynn R. J., Lipsitz L. A., Rockwood K. and Avorn J. (2018). Measuring frailty in medicare data: development and validation of a claims-based frailty index. Gerontol A Biol Sci Med Sci, 73 (7), 980-987.

[11] Walston J., Buta B. and Xue Q. L. (2018). Screening and interventions: considerations for clinical practice. Clin Geriatr Med, 34 (1): 25-38.

[12] Clegg A., Young J., Iliffe S., Rikkert M. O. and Rockwood K. (2013). Frailty in Older People. Lancet, 381 (9868), 752-762.

[13] Rockwood K., Stadnyk K., MacKnight C., McDowell L., Hebert R. and Hogan D. B. (1999). A brief clinical instrument to classify frailty in elderly people. Lancet, 353, 205-206.

[14] Rockwood K. (2005). Frailty and its definition: a worthy challenge. J Am Geriatr Soc, 53 (6), 1069-1070.

[15] Fried L. P., Tangen C. M., Walston J., Newman A. B., Hirsch C., Gottdiener J., Seeman T., Tracy R., Kop W. J., Burke G. and McBurnie M. A. (2001). Frailty in old adults: evidence for a phenotype. J Gerontol A Biol Sci Med Sci, 56 (3), M146156. 